\title{
Color Based Image Retrieval System
}

\author{
Pawandeep Kaur ${ }^{1}$, Sakshi Thakral ${ }^{2}$, Mandeep Singh \\ ${ }^{I}$ (Computer Science Department,Bhai Maha Singh College of Engineering, India) \\ ${ }^{2}$ (Computer Science Department,Bhai Maha Singh College of Engineering, India \\ (Computer Science Department,Bhai Maha Singh College of Engineering, India)
}

\begin{abstract}
Advances in the data storage and image acquisition technologies have enabled the creation of large datasets. It is necessary to develop appropriate information systems to efficiently manage these collections. The most common approaches use Color-Based Image Retrieval (CBIR) system. The goal of CBIR system is to support image retrieval based on color. In a color based image retrieval system querying can be done by a query image. The goal is to find the images most resembling the query. In this work we mainly focused on color histogram-based method.
\end{abstract}

Keywords - Color based image retrieval system (CBIR), Color histogram, Query image

\section{INTRODUCTION}

With the advent of digital photography an ever increasing number of digital images are being produced. Every individual company or agency need to organize their images in any manner. Most easy way to organize images is to categorizing them but this approach has shortcomings like different people might categorize the same image differently and this method is also time consuming [3]. When we deal with very large database we can solve these problems by using color based image retrieval (CBIR), in which image content is used to automatically generate image descriptions when images are searched in large database for extracting the similar content, is known as query by image color[1].This paper is organized as follows. Section 2 shows the requirements for CBIR system. Section 3 is focused on image retrieval system based on color. Section 4 explains user interactions in CBIR system. Section 5 describes methodology of CBIR. Section 6 shows implementation and section 7 represents conclusion. Section 8 presents acknowledgement.

\section{REQUIREMENTS OF CBIR SYSTEM}

There are four requirements of CBIR system:

\subsection{Technique to obtain the metadata, having primitive features of images}

Metadata contains those primitive features having its own representation like color histograms which are used to present color features.

\subsection{User's query demands evaluated by interfaces used}

A query image is used to being the retrieval process and to achieve user's demand. However user cannot always submit an example image to the retrieval system. CBIR system use interface to choose some primitive features for providing an example image.

\subsection{Methods to compare the similar or different images}

CBIR systems require methods that are based on primitive features to compare the similarities or differences between an example image and all the images in the image collection.

\subsection{Efficient indexing and metadata storage techniques}

For a huge image collection, non-trivial methods are used mostly, so CBIR system provides efficient method to compress the metadata. MPEG-7 standard1 is becoming the most important standard to describe all kinds of metadata for both images and video data [3].

\section{IMAGE RETRIEVAL SYSTEM BASED ON COLOR}

Color is most intuitive feature of an image and to describe colors generally histograms are adopted. Histogram methods have the advantages of speediness, low demand of memory space. Color features are the most important elements enabling human to recognize images. For categorizing images, color features can provide powerful information and they are used for image retrieval, so color based image retrieval is mostly used method. Color features of the images are generally represented by color histograms. Before using color histograms, however, we need to select and quantify a color space model and choose a distance metric. 


\subsection{Color Space}

Colors are commonly defined in three-dimensional color spaces. The color space models are of two types. The hardware-oriented color spaces, including RGB (Red Green Blue), CMY (Cyan Magenta Yellow) and YIQ. The user-oriented color spaces, including HLS (Hue Saturation Luminance), HCV, HSV (Hue Saturation value), HSB, MTM, CIE-LAB, and CIE-LUV. The approximate uniform color spaces include MTM, CIE-LAB, and CIE-LUV.

TABLE 1 Color Model Comparison

\begin{tabular}{|l|l|}
\hline \multicolumn{1}{|c|}{ RGB Color Model } & \multicolumn{1}{|c|}{ HSV Color Model } \\
\hline Uses three primary colors, red, green and blue. & Uses hue, saturation and value. \\
\hline $\begin{array}{l}\text { This model has the advantage of being easy to } \\
\text { extract }\end{array}$ & $\begin{array}{l}\text { Not easy extraction as compare to RGB } \\
\text { model. }\end{array}$ \\
\hline $\begin{array}{l}\text { Each pixel value ranging from 0 to 255 giving a } \\
\text { total of 16777216 different colors. }\end{array}$ & $\begin{array}{l}\text { The hue indicates a given color's color- } \\
\text { type and ranges from 0 to 255. }\end{array}$ \\
\hline
\end{tabular}

\subsection{Color Histograms}

A color histogram is the distribution of color in an image. For digital images, a color histogram represents the number of pixels that have colors in each of a fixed list of color ranges, that span the image's color space.

TABLE 2 Local and Global color histogram comparison

\begin{tabular}{|l|l|}
\hline \multicolumn{1}{|c|}{ Local Color Histogram } & \multicolumn{1}{|c|}{ Global Color Histogram } \\
\hline $\begin{array}{l}\text { Segments the image into blocks and then to obtain a color } \\
\text { histogram for each block. }\end{array}$ & $\begin{array}{l}\text { Represents one whole image with a single } \\
\text { color histogram }\end{array}$ \\
\hline $\begin{array}{l}\text { While comparing two images, distance is calculate using their } \\
\text { histograms, between a region in one image and a region in same } \\
\text { location in the other image. }\end{array}$ & $\begin{array}{l}\text { Using the global color histogram an image } \\
\text { will be encoded with its color histogram. }\end{array}$ \\
\hline $\begin{array}{l}\text { The distance between the two images will be determined by the } \\
\text { sum of all these distances. }\end{array}$ & $\begin{array}{l}\text { The distance between two images will be } \\
\text { determined by the distance between their } \\
\text { color histograms. }\end{array}$ \\
\hline
\end{tabular}

\section{II.3 Color Histogram Distance Metrics}

To calculate the distance between color histograms, a variety of metrics have been proposed. We can categorize distance metrics into three ways, namely Minkowski-form distance, Quadratic-form distance, and Non histogram distance. We can define color histograms as a set of bins where each bin denotes the probability of pixels in the image being of a particular color[2].

II.3.1 Minkowski-form distance metrics

Minkowski-form distance metrics compare only the same bins between color histograms.

\section{II.3.2 Quadratic-form distance metrics}

The QBIC project used the Quadratic-form distance metric, which compares not only the same bins but multiple bins between color histograms .Quadratic-form distance metrics overcome a shortcoming of the Minkowski-form distance metrics in that the latter assumes that bins in color histograms are totally unrelated, while the former does not.

\subsubsection{Non-histogram distance metrics}

In this approach, color distribution features of images are represented by their dominant features (termed moments), namely average, variance, and skewness. The first moment is the average color of the image, 
the second is the standard deviation of each color channel, and the third is the third root of each color channel[4].

\section{USER INTERACTION}

Two main features of user interaction in CBIR system is as follows:

\subsection{Query Specification}

Various types of queries can be listed as simple visual feature query, feature combination query, localized feature query, query by example, user-defined attribute query, object relationship query, and concept queries. User can have two ways to make distinction. I first way user looks for category search and in second method he searches for target search.

\subsection{Relevance Feedback}

To improve efficiency and effectiveness of retrieval system relevance feedback(RF) method is adopted.

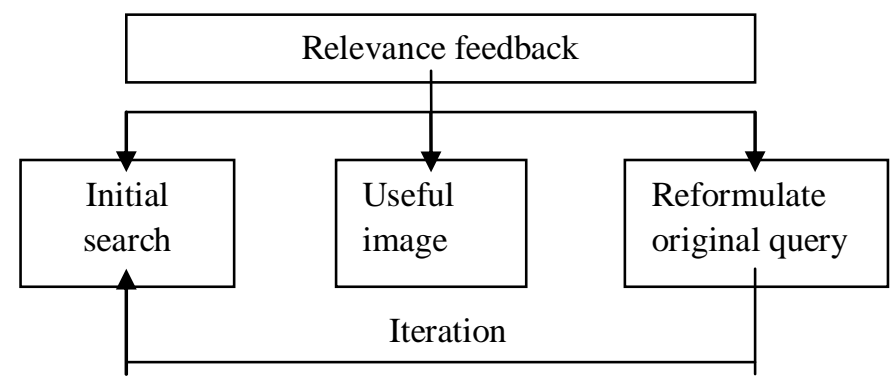

Fig. 1 Flow of relevance feedback

\section{METHODOLOGY}

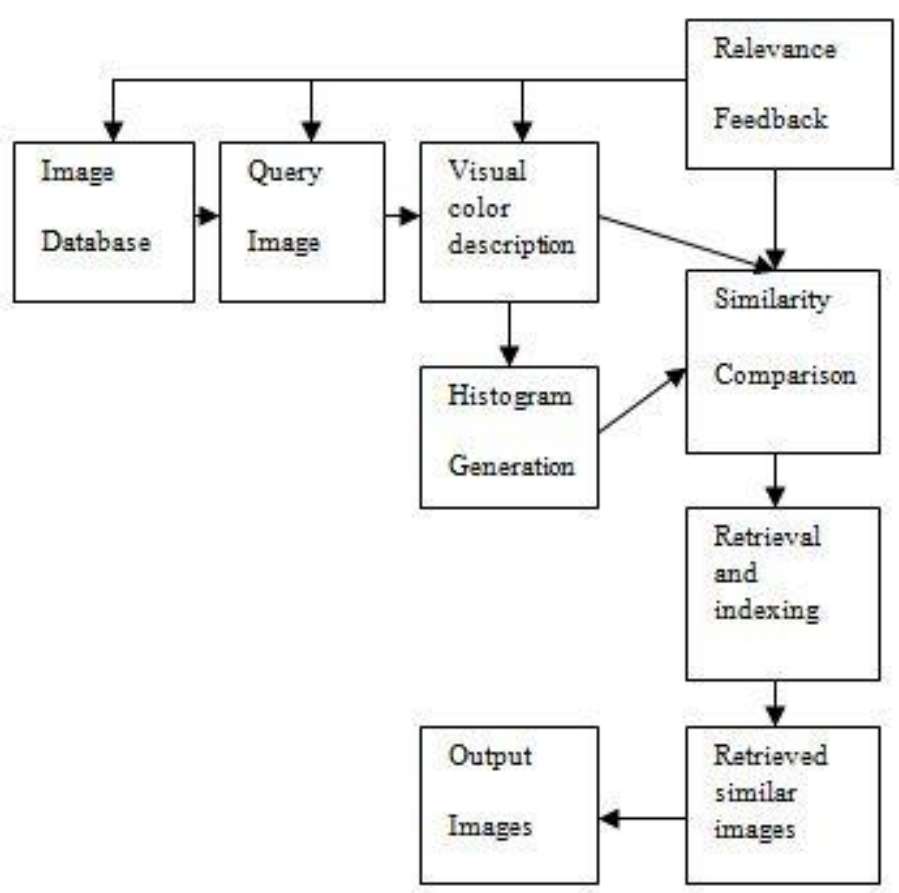

Fig. 2 Block Diagram of CBIR system

\section{IMPLEMENTATION}

Above methodology has been implemented on the image database and one query image is chosen for getting images having almost same histogram. HSV histogram is used for comparison. Here steps of implementation have been shown. 


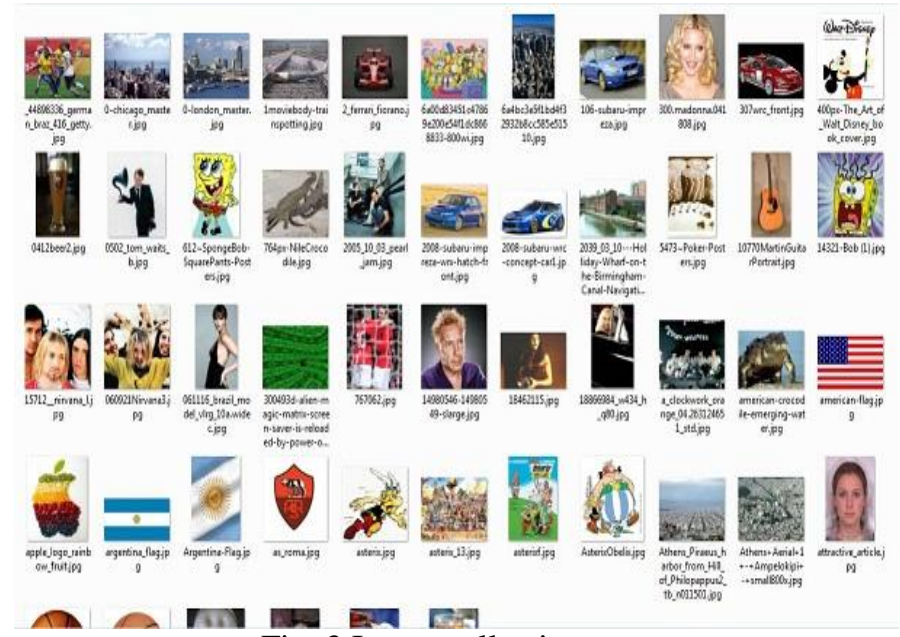

Fig. 3 Image collection

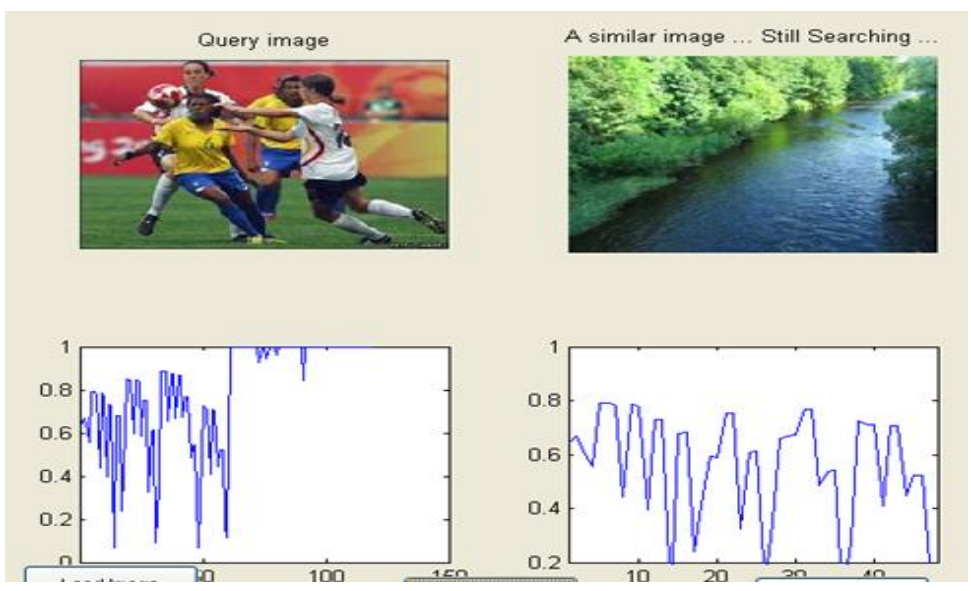

Fig. 4 Images get compared and their equivalent HSV histograms

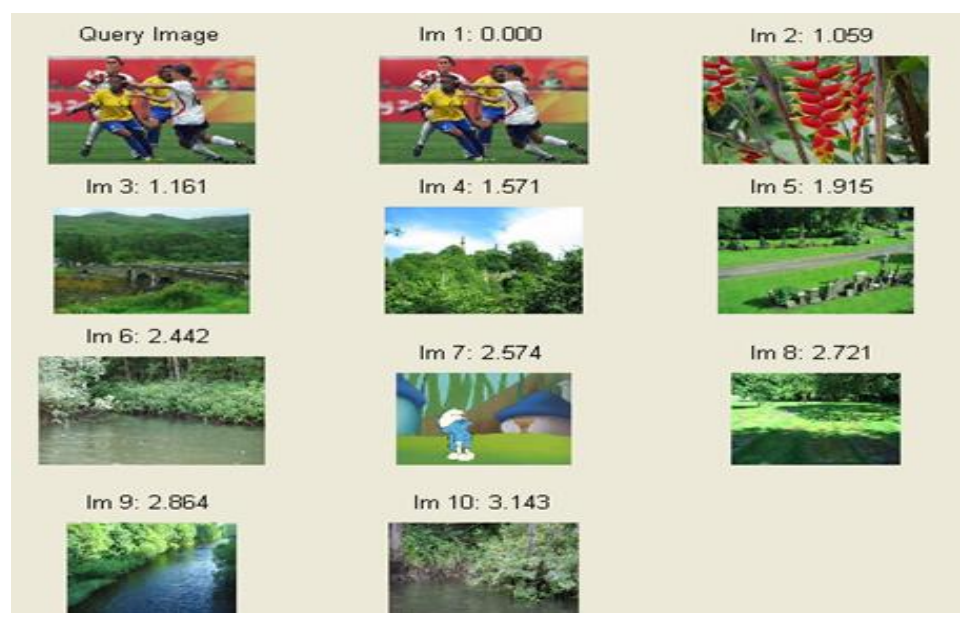

Fig. 5 Ten most resembling images with the query image

\section{CONCLUSION}

Similarity distances are calculated in many ways. Minkowski and Quadratic form distance are the most commonly used distances for image retrieval. By getting relevance feedback of user we can refine query results. In color based image retrieval system, relevance feedback can provide a provision of filling the gap between semantic searching and low-level data processing. But only one low-level aspect of image property is being considered so more improvements are required in this field. 


\section{VIII.}

ACKNOWLEDGEMENTS

Authors are greatly thankful to Dr. Vikas Chawla, Dr. Gurbhinder Singh Brar for useful discussion related to work and useful suggestions.

\section{REFERENCES}

\section{Journal Papers:}

[1] P.S.Suhasini ,Dr. K.Sri Rama Krishna, Dr. I. V. Murli Krishna, CBIR using color hitogram processing, Journal of Theoretical and Applied Information Technology 6(1), $116-122$

[2] Neetu Sharma, Paresh Rawat and jaikaran Singh, Efficient CBIR Using Color Histogram Processing, Signal \& Image Processing , An International Journal(SIPIJ) ,2(1), 2011

Theses:

[3] Ole Andreas Flaaten Jonsgård, Improvements on colour histogram based CBIR, Department of Computer Science and Media Technology Gjøvik University College, 2005

[4] Shengjiu Wang, A Robust CBIR Approach Using Local Color Histograms, University of Alberta, October 2001. 